\title{
Antiplatelet resistance and the role of associated variables in stable patients treated with stenting
}

\author{
Fatih Uzun ${ }^{1}$ Ismail Biyik², Ibrahim Faruk Akturk ${ }^{1}$, Mehmet Erturk ${ }^{1}$, Ahmet Arif Yalcin ${ }^{1}$, Ozgur Surgit ${ }^{1}$, \\ Ender Oner ${ }^{1}$, Hamdi Pusuroglư ${ }^{1}$, Ali Birand ${ }^{1}$ \\ ${ }^{1}$ Department of Cardiology, Mehmet Akif Ersoy Thoracic and Cardiovascular Surgery Training and Research Hospital, Istanbul, Turkey \\ 2Department of Cardiology, Usak State Hospital, Usak, Turkey
}

Postep Kardiol Inter 2015; 11, 1 (39): 19-25 DOI: $10.5114 /$ pwki.2015.49180

\begin{abstract}
A bstract
Introduction: Nowadays, clopidogrel and acetylsalicylic acid (ASA) have become routinely applied therapies in percutaneous coronary interventions ( $\mathrm{PCl}$ ) with stenting.

Aim: Numerous variables can interfere with antiplatelet responsiveness, so we aimed to investigate the role of different variables associated with ASA or clopidogrel resistance in stable coronary artery disease.

Material and methods: A total of 207 patients undergoing elective $\mathrm{PCl}$ were included in the analysis. All patients received a loading dose of clopidogrel and ASA during PCI procedure and followed by dual antiplatelet therapy. Clopidogrel and ASA resistance were measured by impedance aggregometry method.

Results: Of the patients, 19.8\% had clopidogrel resistance, 18.8\% had ASA resistance, 9.2\% had both clopidogrel and ASA resistance, and $71.5 \%$ were responsive to both drugs. In multivariate analysis, platelet count, angiotensin receptor blocker (ARB) use, and ASA resistance were independent variables associated with clopidogrel resistance, and clopidogrel resistance was the only variable associated with ASA resistance. In differentiating whether clopidogrel resistance exists or not, optimum ASA aggregometry response cut-off values were specified, and in differentiating whether ASA resistance exists or not, optimum clopidogrel aggregometry response cut-off values were specified.

Conclusions: In this study, there was a higher incidence of low responsiveness to ASA when there was a low response to clopidogrel, and vice versa. Angiotensin receptor blocker use, platelet count, and ASA resistance were independent variables associated with clopidogrel resistance. Clopidogrel resistance was the only independent variable associated with ASA resistance. Angiotensin receptor blocker use seems to an independent risk factor for clopidogrel resistance in this study, but this result needs to be verified in other studies.
\end{abstract}

Key words: clopidogrel, acetylsalicylic acid, resistance, platelets, angiotensin receptor blocker.

\section{Introduction}

Nowadays, clopidogrel and acetylsalicylic acid have become routinely applied therapies in patients undergoing percutaneous coronary intervention $(\mathrm{PCl})$ with stenting. Current guidelines for coronary procedures recommend dual antiplatelet therapy as a loading dose of clopidogrel and acetylsalicylic acid (ASA) prior to the procedure and the maintenance of dual antiplatelet therapy with clopidogrel and ASA to reduce thrombotic events [1]. However, there is considerable individual heterogeneity in the responses to these drugs between patients. Reduced platelet inhibition is an important factor in worse clinical outcomes in patients treated with clopidogrel and/or ASA. Previous studies have reported that adequate antiplatelet effects of ASA are not accomplished in $5 \%$ to $45 \%$ of patients with coronary artery disease (CAD) [2-5]. The incidence of low response or non-response to clopidogrel ranges from $4 \%$ to $30 \%$ of patients with cardiovascular disease [6-8]. The lower response to clopidogrel among aspirin-resistant patients is of particular clinical importance because clopidogrel has been suggested as an alternative therapy for aspirin-re-

\section{Corresponding author:}

Ismail Biyik MD, Asc. Prof., Uşak Devlet Hastanesi Kardiyoloji Kliniği: 64100 Uşak, Turkiye, phone: +905424173209,

e-mail: ismailbiyikmd@yahoo.com

Received: 17.03.2014, accepted: 5.05.2014. 
sistant patients. It has been reported that $47 \%$ of aspirin-resistant patients are also clopidogrel resistant [9]. Approximately $10 \%$ of patients undergoing $\mathrm{PCl}$ are poor responders to both aspirin and clopidogrel [10]. The wide range in platelet resistance between different studies has not been clarified well. This wide range is attributed to clinical differences in the case mix of the patients, different doses of antiplatelets used, and the differences in the methodology used to assess responsiveness to antiplatelet therapy [11]. There are no well-defined criteria or parameters predicting antiplatelet resistance. Moreover, there is a lack of laboratory or clinical parameters to define which patients are most likely benefit from platelet function studies. In recent years, haematological parameters such as the red blood cell distribution width (RDW), mean platelet volume (MPV), and neutrophil/lymphocyte ratio $(\mathrm{N} / \mathrm{L})$ have frequently been used to assess cardiovascular risk [12-14]. Numerous variables such as patients' medicines and haematological variables might interfere with antiplatelet responsiveness. The value of these parameters in determining ASA or clopidogrel resistance is not well known.

\section{Aim}

In the present study, we aimed to investigate the role of different variables associated with ASA or clopidogrel resistance in patients with stable coronary artery disease treated with stenting.

\section{Material and methods}

\section{Study population}

This is an observational single-centre study of 221 consecutive patients with stable coronary artery disease undergoing elective $\mathrm{PCl}$ in a tertiary heart centre. Complete medical history including risk factors, concomitant diseases, and medications was taken for all patients. The study protocol was approved by the Ethics Committee of the institution, and all patients gave written informed consent for participation. Patients who were given bare metal coronary stents in the preceding month were included in the study. All patients received a loading dose of 600 mg clopidogrel and 300 mg ASA during or before PCl procedure followed by regular dual antiplatelet therapy with $100 \mathrm{mg}$ ASA and $75 \mathrm{mg}$ clopidogrel, daily. Other medications of the patients such as diuretics, statins, $\beta$-blockers, nitrates, and anti-hypertensive drugs were continued in the same order. Laboratory low responsiveness to ASA and clopidogrel were measured and their relationships with several important clinical and laboratory variables were analysed. The exclusion criteria of the study were any history of bleeding abnormalities, use of any other antiplatelet drugs, anticoagulants or non-steroid anti-inflammatory drugs, any history of drug-induced thrombocytopaenia, coronary bypass grafting during the preced- ing 3 months, contraindications to antiplatelet therapy, platelet count $<100,000$ cells $/ \mathrm{mm}^{3}$, presence of significant anaemia, hepatic or renal disturbances, connective tissue disease, neoplastic or inflammatory disease, and poor adherence to antiplatelet therapy with clopidogrel and ASA. Fourteen patients were also excluded later due to incomplete data.

\section{Blood sampling}

Baseline laboratory tests were performed in the period of hospital admittance. Laboratory tests included complete blood count, neutrophil/lymphocyte ratio, lipid profile, fasting blood glucose, glycated haemoglobin $\left(\mathrm{HbA}_{1 \mathrm{c}}\right)$, and kidney and liver function tests. Parameters were assessed again at 1 month after hospital discharge during clinical follow-up. Venous blood samples from all the patients receiving clopidogrel and/or aspirin were collected in test tubes containing hirudin.

\section{Platelet aggregation testing}

Clopidogrel and aspirin resistances were measured by impedance aggregometry method with a multiple electrode aggregometry device (Multiplate, Dynabyte Medical, Munich, Germany). Impedance aggregometry measures electrical impedance after exposure to whole blood suspension by a platelet agonist [15]. The impedance aggregometry method is based on the principle that platelets are non-thrombogenic in the resting phase; however, when activated they uncover receptors on their surfaces which help them adhere to vessel walls and artificial surfaces. Platelets increase the resistance through the sensor when bound to sensor wires in the activator-added multiplate test bathtubs. The increased impedance due to platelets adhered to multiplate sensors is converted into aggregation units (AU) and an AU-versus-time graphic is created. Clopidogrel and aspirin low responsiveness are evaluated by area under the curve (AUC). The major advantage of this method is that it takes into account the interactions between platelets with the other blood cellular elements [16].

\section{Definition of antiplatelet resistance}

There is no universally accepted definition of ASA or clopidogrel resistance. Laboratory ASA resistance is defined as the inability of therapeutic doses of ASA to inhibit platelet aggregation, which is a primary measurement of platelet function or a failure to reduce TXA ${ }_{2}$ [17]. The incapacity of clopidogrel to inhibit platelet aggregation measured at the laboratory is known as clopidogrel resistance [18]. As Sibbing et al. defined a cut-off point at the upper quintile as clopidogrel low responsiveness, and the most widely used definition of ASA resistance is a > 20\% aggregometry response to the platelet agonist $[19,20]$, we also defined clopidogrel or ASA resistance as patients with a high on-treatment platelet aggregation 
above the fifth quartile with a value $\geq 20 \%$ : $601.8 \mathrm{AU} /$ min for clopidogrel and $447.6 \mathrm{AU} / \mathrm{min}$ for aspirin.

\section{Statistical analysis}

Continuous variables were expressed as mean \pm SD. Categorical variables were compared using $\chi^{2}$ test. Student's $t$-test was used for normally distributed continuous variables. Multiple logistic regression analyses with enter method were performed to identify the independent risk factors associated with clopidogrel/ASA resis- tance. Statistical analyses were performed using SPSS 19.0 (Chicago, IL, USA). Statistical significance was assumed at $p<0.05$.

\section{Results}

In total 207 patients were included in the analysis. Eighty-three percent of patients were male, mean age was $55.5 \pm 9.7$ years, and mean body mass index (BMI) was $28.2 \pm 4.2 \mathrm{~kg} / \mathrm{m}^{2}$. Of the patients, $19.8 \%(n=41)$ had clopidogrel resistance, $18.8 \%(n=39)$ had ASA re-

Table I. Univariate analyses of clopidogrel and ASA resistances

\begin{tabular}{|c|c|c|c|c|c|c|}
\hline \multirow[t]{2}{*}{ Characteristics } & \multicolumn{2}{|c|}{ Clopidogrel resistance } & \multirow[t]{2}{*}{ Value of $p$} & \multicolumn{2}{|c|}{ ASA resistance } & \multirow[t]{2}{*}{ Value of $p$} \\
\hline & $\begin{array}{c}\text { Absent } \\
(n=166)\end{array}$ & $\begin{array}{l}\text { Present } \\
(n=41)\end{array}$ & & $\begin{array}{c}\text { Absent } \\
(n=168)\end{array}$ & $\begin{array}{l}\text { Present } \\
(n=39)\end{array}$ & \\
\hline Age [years] & $55.6 \pm 9.6$ & $54.9 \pm 10.3$ & 0.680 & $55.1 \pm 9.5$ & $56.7 \pm 10.8$ & 0.366 \\
\hline Male sex (\%) & 16.3 & 37.1 & 0.009 & 17.7 & 31.2 & 0.092 \\
\hline Smoker (\%) & 19 & 17 & 0.832 & 17.4 & 23.3 & 0.380 \\
\hline $\mathrm{BMI}\left[\mathrm{kg} / \mathrm{m}^{2}\right]$ & $27.9 \pm 4$ & $29.4 \pm 4.8$ & 0.053 & $28.1 \pm 4.1$ & $28.8 \pm 4.6$ & 0.409 \\
\hline ASA resistance (\%) & 12.7 & 48.7 & $<0.0001$ & - & - & - \\
\hline \multicolumn{7}{|l|}{ Biochemical parameters: } \\
\hline $\mathrm{HDL}[\mathrm{mg} / \mathrm{dl}]$ & $40 \pm 9.4$ & $41.2 \pm 9.2$ & 0.449 & $40 \pm 9.2$ & $40.6 \pm 10.6$ & 0.756 \\
\hline Total cholesterol [mg/dl] & $169.9 \pm 42.7$ & $166.6 \pm 39.1$ & 0.653 & $163.9 \pm 41$ & $188.8 \pm 42$ & 0.001 \\
\hline Triglycerides [mg/dl] & $167.6 \pm 120.2$ & $152.9 \pm 74.3$ & 0.457 & $165.8 \pm 120.2$ & $162.7 \pm 91.3$ & 0.882 \\
\hline VLDL [mg/dl] & $30.4 \pm 14.6$ & $29.6 \pm 12.8$ & 0.778 & $30.1 \pm 14.6$ & $30.3 \pm 13.5$ & 0.965 \\
\hline $\mathrm{LDL}[\mathrm{mg} / \mathrm{dl}]$ & $101.9 \pm 35.8$ & $99.2 \pm 31.2$ & 0.655 & $97.3 \pm 34.1$ & $114.7 \pm 35.2$ & 0.005 \\
\hline Blood glucose [mg/dl] & $122.2 \pm 51.8$ & $130.1 \pm 63.4$ & 0.405 & $123 \pm 54.1$ & $128.5 \pm 60.3$ & 0.579 \\
\hline $\mathrm{HbA}_{1 \mathrm{c}}(\%)$ & $6.3 \pm 1.3$ & $6.5 \pm 1.4$ & 0.402 & $6.4 \pm 1.4$ & $6.6 \pm 1.3$ & 0.429 \\
\hline Creatinine $[\mathrm{mg} / \mathrm{dl}]$ & $0.94 \pm 0.24$ & $0.87 \pm 0.24$ & 0.086 & $0.93 \pm 0.24$ & $0.88 \pm 0.21$ & 0.181 \\
\hline Uric acid [mg/dl] & $5.6 \pm 1.4$ & $5.3 \pm 1.5$ & 0.280 & $5.6 \pm 1.4$ & $5.4 \pm 1.4$ & 0.458 \\
\hline GFR $\left[\mathrm{ml} / \mathrm{min} / 1.73 \mathrm{~m}^{2}\right]$ & $89.3 \pm 18.8$ & $91.8 \pm 3.4$ & 0.454 & $89.2 \pm 18.8$ & $92.6 \pm 20.4$ & 0.329 \\
\hline \multicolumn{7}{|l|}{ Hematologic parameters: } \\
\hline WBC $\left[10^{3} / \mathrm{mm}^{3}\right]$ & $7739 \pm 1913$ & $8202 \pm 2198$ & 0.179 & $7782 \pm 1961$ & $8101 \pm 2232$ & 0.378 \\
\hline Haemoglobin [g/dl] & $14.2 \pm 1.4$ & $13.0 \pm 2.0$ & $<0.0001$ & $14.1 \pm 1.5$ & $13.4 \pm 2.0$ & 0.03 \\
\hline Haematocrit (\%) & $42.4 \pm 5.6$ & $39.4 \pm 5.5$ & 0.002 & $42.2 \pm 5.8$ & $40.6 \pm 5.7$ & 0.128 \\
\hline Platelet count $\left[10^{3} / \mathrm{mm}^{3}\right]$ & $235.9 \pm 56.6$ & $298.3 \pm 131$ & $<0.0001$ & $238.2 \pm 58.2$ & $290.5 \pm 133.5$ & $<0.0001$ \\
\hline MPV $[\mathrm{fl}]$ & $8.6 \pm 0.9$ & $8.7 \pm 0.9$ & 0.633 & $8.6 \pm 0.9$ & $8.7 \pm 0.9$ & 0.462 \\
\hline RDW (\%) & $13.3 \pm 1.3$ & $13.8 \pm 1.8$ & 0.031 & $13.3 \pm 1.3$ & $13.6 \pm 1.8$ & 0.234 \\
\hline N/L ratio & $2.2 \pm 1$ & $2.5 \pm 0.9$ & 0.124 & $2.2 \pm 1.0$ & $2.3 \pm 0.8$ & 0.740 \\
\hline \multicolumn{7}{|l|}{ Medications: } \\
\hline$\beta$-Blockers (\%) & 25 & 17.9 & 0.352 & 34.4 & 16.7 & 0.028 \\
\hline Diuretics (\%) & 18.2 & 22 & 0.219 & 19.2 & 24.1 & 0.614 \\
\hline Statin (\%) & 19.2 & 20.1 & 1.000 & 25 & 17.2 & 0.195 \\
\hline Nitrate (\%) & 20.2 & 17.6 & 0.818 & 19.8 & 20.7 & 1.000 \\
\hline ACEI (\%) & 24.5 & 15 & 0.116 & 21.8 & 18.1 & 0.592 \\
\hline ARBs (\%) & 16.3 & 41.4 & 0.004 & 18.8 & 26.9 & 0.427 \\
\hline CCB (\%) & 18.8 & 26.9 & 0.428 & 17.2 & 40.9 & 0.019 \\
\hline Trimetazidine (\%) & 19.3 & 25 & 0.557 & 19.1 & 27.8 & 0.363 \\
\hline Sulfonylurea (\%) & 18.8 & 26.9 & 0.428 & 18.1 & 32 & 0.113 \\
\hline Insulin (\%) & 20 & 16.7 & 1.000 & 19.5 & 33.3 & 0.342 \\
\hline
\end{tabular}

Data are presented as mean \pm SD or $n(\%)$. ASA - acetylsalicylic acid, ACEI - angiotensin-converting enzyme inhibitors, ARBS - angiotensin II receptor blockers, BMI - body mass index (kg/m2), CCB - calcium channel blockers, GFR-glomerular filtration rate (ml/min/1.73 m2), HbAlc-haemoglobin A1c, HDL - high-density lipoprotein, MPV - mean platelet volume, $L D L$ - low-density lipoprotein, RDW-red cell distribution width, $N / L$-neutrophil/lymphocyte, VLDL - very low density lipoprotein. 
sistance, 9.2\% ( $n=19)$ had both clopidogrel and ASA resistance, and $61.4 \%(n=127)$ were responsive to both drugs. The groups did not differ in erythrocyte counts. However, platelet counts and haemoglobin levels were higher in responders (Table I).

\section{Clopidogrel resistance}

In univariate analyses, clopidogrel resistance was associated with male sex, higher BMI, ASA resistance, lower haemoglobin and haematocrit levels, higher RDW levels, higher platelet counts, and angiotensin II receptor blocker (ARB) use (Table I). Higher platelet count $(\mathrm{OR}=1.009 ; 95 \% \mathrm{Cl}: 1.001-1.016)$, ARB use $(\mathrm{OR}=4.29$; 95\% Cl: 1.44-12.76), and ASA resistance (OR $=4.79$; 95\% Cl: 1.9-12.1) were independent variables associated with clopidogrel resistance in multivariate analysis (Table II). The discriminative value of ASA resistance in differentiating whether clopidogrel resistance exists was AUC 0.768, SE (Std. error) 0.045 (95\% Cl: 0679-0.857) and $p$-value $<0.0001$. For differentiation of whether clopidogrel resistance exists or not, optimum 'ASA aggregometry response' cut-off values are shown in Table III.

\section{Acetylsalicylic acid resistance}

Univariate analyses showed that higher total cholesterol and LDL levels, lower haemoglobin levels, higher platelet counts, clopidogrel resistance, and use of $\beta$-blockers or calcium channel blockers (CCB) were associated with ASA resistance (Table I). Clopidogrel resistance (OR $=5.8 ; 95 \% \mathrm{Cl}: 2.18-15.45)$ was the only variable associated with ASA resistance, according to multivariate analysis. The discriminative value of clopidogrel resistance in differentiating whether ASA resistance exists was AUC 0.763, SE 0.046 (95\% Cl: 0674-0.853), and p-value $<0.0001$. For differentiation of whether ASA resistance exists or not, optimum 'clopidogrel aggregometry response' cut-off values are shown in Table IV.

\section{Discussion}

This study revealed that high platelet count, angiotensin II receptor blocker use, and ASA resistance were independent variables associated with clopidogrel resistance, and clopidogrel resistance was the only independent variable associated with ASA resistance. The most interesting result of this study was that angiotensin II

Table II. Multivariate analysis of clopidogrel/ASA resistance

\begin{tabular}{|c|c|c|c|c|c|}
\hline Variables & $\beta$ & Standard error & Value of $p$ & OR & $95 \% \mathrm{Cl}$ \\
\hline \multicolumn{6}{|l|}{ Clopidogrel resistance: } \\
\hline Gender (M/F) & -0.485 & 0.572 & 0.397 & 0.62 & $0.20-1.89$ \\
\hline $\mathrm{BMI}\left[\mathrm{kg} / \mathrm{m}^{2}\right]$ & 0.039 & 0.052 & 0.453 & 1.04 & $0.94-1.15$ \\
\hline Creatinine $[\mathrm{mg} / \mathrm{dl}]$ & -1.283 & 1.189 & 0.280 & 0.28 & $0.03-2.85$ \\
\hline Haematocrit (\%) & -0.044 & 0.055 & 0.431 & 0.96 & $0.86-1.07$ \\
\hline RDW (\%) & 0.09 & 0.177 & 0.613 & 1.094 & $0.77-1.55$ \\
\hline Platelet count $\left[10^{3} / \mathrm{mm}^{3}\right]$ & 0.009 & 0.004 & 0.021 & 1.009 & $1.001-1.016$ \\
\hline ARBs use (yes/no) & 1.456 & 0.556 & 0.009 & 4.29 & $1.44-12.76$ \\
\hline ASA resistance (yes/no) & 1.566 & 0.473 & 0.001 & 4.79 & $1.9-12.1$ \\
\hline \multicolumn{6}{|l|}{ ASA resistance: } \\
\hline Gender (M/F) & -0.17 & 0.595 & 0.775 & 0.84 & $0.26-2.71$ \\
\hline Clopidogrel resistance (yes/no) & 1.759 & 0.499 & $<0.0001$ & 5.8 & $2.18-15.45$ \\
\hline Total cholesterol [mg/dl] & 0.014 & 0.012 & 0.223 & 1.01 & $0.99-1.04$ \\
\hline $\mathrm{LDL}[\mathrm{mg} / \mathrm{dl}]$ & 0.005 & 0.014 & 0.733 & 1.01 & $0.98-1.03$ \\
\hline Haemoglobin [g/dl] & -0.12 & 0.148 & 0.415 & 0.89 & $0.66-1.18$ \\
\hline Platelet count $\left[10^{3} / \mathrm{mm}^{3}\right]$ & 0.006 & 0.003 & 0.061 & 1.01 & $1.00-1.012$ \\
\hline$\beta$-Blockers (yes/no) & 0.719 & 0.520 & 0.167 & 2.1 & $0.74-5.69$ \\
\hline CCB (yes/no) & -0.971 & 0.583 & 0.096 & 0.38 & $0.12-1.19$ \\
\hline
\end{tabular}

$A S A$ - acetylsalicylic acid, ARBs - angiotensin II receptor blockers, BMI - body mass index ( $\left.\mathrm{kg} / \mathrm{m}^{2}\right), C C B$ - calcium-channel blockers, $L D L$ - low-density lipoprotein, $R D W$ - red cell distribution width.

Table III. Diagnostic value of cut-off value of ASA aggregometry response for defining clopidogrel resistance

\begin{tabular}{lccccc}
$\begin{array}{l}\text { Cut-off value of ASA aggregometry response } \\
\text { [AU/min] as a prognostic marker }\end{array}$ & Sensitivity (\%) & Specificity (\%) & LR+ & LR- \\
\hline 258.5 & 87.2 & 37.6 & 1.4 & 0.34 \\
\hline 627.5 & 41 & 90.4 & 4.3 & 0.65 \\
\hline 739.0 & 23.1 & 95.5 & 5.2 & 0.81
\end{tabular}


Table IV. Diagnostic value of cut-off value of clopidogrel aggregometry response for defining ASA resistance

\begin{tabular}{cccccc}
$\begin{array}{l}\text { Cut-off value of clopidogrel aggregometry response } \\
\text { [AU/min] as a prognostic marker }\end{array}$ & Sensitivity (\%) & Specificity (\%) & LR+ & LR- \\
\hline 171.0 & 84.6 & 44.6 & 1.5 & 0.35 \\
\hline 588.5 & 43.6 & 90.4 & 4.6 & 0.62 \\
\hline 693.5 & 41 & 94.3 & 7.2 & 0.63
\end{tabular}

receptor blocker use seemed to be an independent risk factor for clopidogrel resistance. This study also demonstrated that haematological parameters such as RDW, $M P V$, and N/L ratio did not predict antiplatelet resistance. Previous studies examining resistance to clopidogrel and associated variables gave different results. Genetic predispositions, female gender, older age, blood glucose level, diabetes, and high systolic and diastolic blood pressure were correlated with an ineffective clopidogrel response [11, 21-23]. In the multivariate logistic regression analysis of the recent study by Osmancik et al., older age, higher weight, female gender, mechanical ventilation and higher concentration of leukocytes and interleukin 10 (IL-10) were associated with an increased risk for being a non-responder to clopidogrel [24]. In another recent study by Lundström et al., clinical conditions found to be associated with high on-treatment platelet reactivity were diabetes mellitus, high body-mass index, acute coronary syndrome, left ventricular failure, and chronic renal failure [25]. In univariate analyses, the present study revealed that male sex, higher BMI, ASA resistance, lower haemoglobin and haematocrit levels, higher platelet count, higher RDW levels, and ARB use were associated with clopidogrel resistance, but high platelet count, ARB use, and ASA resistance were independent variables related to clopidogrel resistance in multivariate analysis. Previously, higher responders to clopidogrel among smokers (the so-called smoking paradox) have been described by several authors [24-26]. However, in the present study, there was no association with smoking habit and clopidogrel/ASA response. According to our knowledge, the present study might be the first study drawing attention to the fact that ARB use might be an independent risk factor for clopidogrel resistance. As a consequence of angiotensin II receptor (AT2) blockade, ARBs increase angiotensin II levels several fold above baseline by uncoupling the negative feedback loop. An increased level of circulating angiotensin II results in unopposed stimulation of the AT2 receptors, which are up-regulated in this case. Recent data suggest that AT2 receptor stimulation may be harmful under certain circumstances, through mediation of growth promotion, fibrosis, and hypertrophy as well as proatherogenic and proinflammatory effects [27]. Ge et al. pointed out that clopidogrel resistance is associated with systemic inflammation [28]. In the present study, the relationship between ARB use and clopidogrel resistance may be at least partly associated with increased inflammatory process caused by long-term ARB use. Conversely, in patients taking angiotensin converting enzyme (ACE) inhibitors, no higher resistance to clopidogrel was found in this study, supporting the theory that the pathophysiological processes may be due to pharmacodynamics of ARB and not to ACE inhibitors. In the National Swedish Stroke Registry, ARB use did not differ between responders and non-responders to clopidogrel, but use of CCBs was statistically higher in non-responders to clopidogrel [25]. The CCBs used were all dihydropyridines such as amlodipine and felodipine. Conversely, no difference was found in another study [19]. Knight et al. tried to explain the effect of CCBs on high platelet resistance as drug-drug interactions with clopidogrel via CYP3A4, or due to the platelet activating effect of CCBs [29]. Investigations of factors affecting aspirin resistance have given different results, probably due to clinical differences of the patients, different doses and types of ASA used (e.g. enteric coated), and differences in the laboratory tests used to assess resistance to aspirin therapy. The associated factors with ASA resistance have been described as female sex, increased age, diabetes, high plasma triglycerides, low hemoglobin level, end-stage kidney disease, simultaneous administration of other non-steroidal anti-inflammatory drugs, elevated norepinephrine levels, cigarette smoking, hypercholesterolemia, polymorphisms affecting COX-1 and transient increase of platelet COX-1/ COX-2 expression in new platelets [15-24]. In another review, clinical situations associated with ASA resistance have been asserted as hyperlipidaemia, diabetes mellitus, hypertension, heart failure, and inflammatory disorders [16]. However, the presence of athero-thrombotic risk factors such as hypertension, smoking, family history of ischaemic heart disease, and previous myocardial infarction (MI) were not found to be different between aspirin-resistant and aspirin-sensitive patients in a recent study, but diabetes mellitus and dyslipidaemia did differ between the aspirin-sensitive and the aspirin-resistant patients [30]. In the elderly population, elevated fasting serum glucose level was found as the only significant independent risk factor for aspirin resistance as determined by thromboelastography platelet mapping assay [31]. Abacı and Kiliçkesmez pointed out that the drug interactions were important causes of aspirin resistance and that a higher percentage of aspirin resistance was observed among patients who take statins, non-steroidal anti-inflammatory drugs, and proton pump inhibitors 
[17]. Hobikoglu et al. found an increased sensitivity of platelets to ADP in patients with aspirin resistance [32].

In a univariate analyses of the present study, total cholesterol and LDL levels, lower haemoglobin levels, higher platelet counts, clopidogrel resistance, and use of $\beta$-blockers or CCB were significantly different between aspirin-resistant and aspirin-sensitive patients, but clopidogrel resistance $(\mathrm{OR}=5.8 ; 95 \% \mathrm{Cl}$ : 2.18-15.45) was the only independent variable associated with ASA resistance according to the multivariate analysis of the study. It is known that clopidogrel is less sensitive in patients with aspirin resistance and that some patients have resistance to both drugs [17]. The present study also pointed out sensitive cut-off points defining cross resistance between these two antiplatelet drugs. The results regarding the associations between lipid levels and ASA resistance in this study were similar to the results of previous studies. In a geriatric population in China, aspirin-resistant patients had higher levels of LDL cholesterol compared to aspirin-sensitive patients [31]. Akoglu et al. found that serum LDL cholesterol levels were closely associated with aspirin resistance in 83 patients with nephrotic syndrome [33]. In an extended study of 972 patients, platelet aggregation was correlated with high LDL cholesterol levels [34]. It has also been reported that ASA resistance was associated with decreased HDL levels in patients with acute coronary syndrome [35]. Similarly, Tanrikulu et al. reported that low HDL cholesterol was an independent predictor of aspirin resistance in a cohort of patients with chronic renal failure [36]. The platelet responses to clopidogrel and inflammation seem to be closely related. Osmancik et al. put forward the idea that the elevations of leukocytes and IL-10 levels related to an increased state of inflammation were associated with higher on-treatment platelet reactivity [24]. The present study also investigated the associations between inflammation markers such as RDW level, MPV and N/L ratio, and antiplatelet resistance. Among these parameters, only RDW levels significantly differed between patients with and without clopidogrel resistance in the univariate analysis, but the multivariate logistic regression analysis revealed that RDW, MPV, and N/L ratio were not adequate to identify patients with antiplatelet therapy resistance.

This study has several limitations. Some important variables such as fibrinogen levels and inflammatory state and the use of other medications already associated with clopidogrel resistance such as proton pomp inhibitors were not studied. Tests for aspirin and clopidogrel resistance were not repeated to confirm the drug resistance. Long-term follow up data were not evaluated to reveal the clinical importance of the results. The plasma levels of the drugs were not measured to exclude patients' noncompliance, but we tried to overcome this issue by checking empty blisters of drug boxes. Moreover, genetic screening for CYPD 219 gene was not performed.
The ARB therapy of the patients in which clopidogrel resistance was statistically higher was not changed for re-evaluation of drug resistance because of the observational nature of the study.

\section{Conclusions}

The present study showed that there is higher incidence of non-responsiveness to aspirin when there is low response to clopidogrel, or vice versa. The ARB use, platelet count, and ASA resistance were independent variables associated with clopidogrel resistance. Clopidogrel resistance was the only independent variable associated with ASA resistance. The most interesting result of this study is that angiotensin II receptor blocker use seems to be an independent risk factor for clopidogrel resistance. However, this result should be verified in well-designed, largescale studies on antiplatelet therapy resistance.

\section{Conflict of interest}

The authors declare no conflict of interest.

\section{References}

1. 2011 ACCF/AHA/SCAI Guideline for Percutaneous Coronary Intervention: A Report of the American College of Cardiology Foundation/American Heart Association Task Force on Practice Guidelines and the Society for Cardiovascular Angiography and Interventions. Circulation 2011; 124: e574-651.

2. Buchanan MR, Brister SJ. Individual variation in the effects of ASA on platelet function: implications for the use of ASA clinically. Can J Cardiol 1995; 11: 221-7.

3. Eikelboom JW, Hirsh J, Weitz JI, et al. Aspirin resistant thromboxane biosynthesis and the risk of myocardial infarction, stroke, or cardiovascular death in patients at high risk for cardiovascular events. Circulation 2002; 105: 1650-5.

4. Gum PA, Kottke-Marchant K, Welsh PA, et al. A prospective, blinded determination of the natural history of aspirin resistance among stable patients with cardiovascular disease. J Am Coll Cardiol 2003; 41: 961-5.

5. Gum PA, Kottke-Marchant K, Poggio ED, et al. Profile and prevalence of aspirin resistance in patients with cardiovascular disease. Am J Cardiol 2001; 88: 230-5.

6. Gurbel PA, Bliden KP, Hiatt BL, et al. Clopidogrel for coronary stenting: response variability, drug resistance, and the effect of pretreatment platelet reactivity. Circulation 2003; 107: 2908-13.

7. Serebruany VL, SteinhubI SR, Berger PB, et al. Variability in platelet responsiveness to clopidogrel among 544 individuals. J Am Coll Cardiol 2005; 45: 246-51.

8. Muller I, Besta F, Schulz C, et al. Prevalence of clopidogrel nonresponders among patients with stable angina pectoris scheduled for elective coronary stent placement. Thromb Haemost 2003; 89: 783-7.

9. Lev El, Patel RT, Maresh KJ, et al. Aspirin and clopidogrel drug response in patients undergoing percutaneous coronary intervention: the role of dual drug resistance. J Am Coll Cardiol 2006; 47: 27-33.

10. Lepantalo A, Virtanen KS, Heikkila J, et al. Limited early antiplatelet effect of $300 \mathrm{mg}$ clopidogrel in patients with aspirin 
therapy undergoing percutaneous coronary interventions. Eur Heart J 2004; 25: 476-83.

11. Mijajlovic MD, Shulga O, Bloch S, et al. Clinical consequences of aspirin and clopidogrel resistance: an overview. Acta Neurol Scand 2013; 128: 213-9.

12. Arbel Y, Birati EY, Finkelstein A, et al. Red blood cell distribution width and 3-year outcome in patients undergoing cardiac catheterization. J Thromb Thrombolysis 2014; 37: 469-74.

13. Wan ZF, Zhou D, Xue JH, et al. Combination of mean platelet volume and the GRACE risk score better predicts future cardiovascular events in patients with acute coronary syndrome. Platelets 2014; 25: 447-51.

14. Yalcin M, Aparci M, Uz O, et al. Neutrophil-lymphocyte ratio may predict left atrial thrombus in patients with nonvalvular atrial fibrillation. Clin Appl Thromb Hemost 2015; 21: 166-71.

15. Gorelick PB, Farooq MU. Advances in our understanding of "resistance" to antiplatelet agents for prevention of ischemic stroke. Stroke Res Treat 2013; 2013: 727-842.

16. Kasmeridis C, Apostolakis S, Lip GY. Aspirin and aspirin resistance in coronary artery disease. Curr Opin Pharmacol 2013; 13: 242-50.

17. Abacı O, Kılıçkesmez KO. Aspirin resistance: where are we now? Anadolu Kardiyol Derg 2013; 13: 370-3.

18. Silva FB, Almeida Junior GL, Neno A, et al. Resistance to clopidogrel: prevalence and associate variables. Arq Bras Cardiol 2012; 99: 1135-41.

19. Sibbing D, Braun S, Morath T, et al. Platelet reactivity after clopidogrel treatment assessed with point-of-care analysis and early drug eluting stent thrombosis. J Am Coll Cardiol 2009; 53: 84956.

20. Sibbing D, Morath T, Braun S, et al. Clopidogrel response status assessed with multiplate point-of-care analysis and the incidence and timing of stent thrombosis over six months following coronary stenting. Thromb Haemost 2010; 103: 151-9.

21. Akturk IF, Caglar FN, Erturk M, et al. Hypertension as a risk factor for aspirin and clopidogrel resistance in patients with stable coronary artery disease. Clin Appl Thromb Hemost 2014; 20: 749-54.

22. Marcucci R, Gori AM, Paniccia R, et al. Cardiovascular death and non-fatal myocardial infarction in acute coronary syndrome patients receiving coronary stenting are predicted by residual platelet reactivity to ADP detected by a point-of-care assay: a 12 month follow-up. Circulation 2009; 119: 237-42.

23. Price MJ, Endemann S, Gollapudi RR, et al. Prognostic significance of post-clopidogrel platelet reactivity assessed by a pointof-care assay on thrombotic events after drug-eluting stent implantation. Eur Heart J 2008; 29: 992-1000.

24. Osmancik P, Paulu P, Tousek P, et al. High leukocyte count and interleukin-10 predict high on-treatment-platelet-reactivity in patients treated with clopidogrel. J Thromb Thrombolysis 2012; 33: 349-54

25. Lundström A, Laska AC, Von Arbin M, et al. Glucose intolerance and insulin resistance as predictors of low platelet response to clopidogrel in patients with minor ischemic stroke or TIA. Platelets 2014; 25: 102-10.

26. Motovska Z, Widimsky P, Petr R, et al. Factors influencing clopidogrel efficacy in patients with stable coronary artery disease undergoing elective percutaneous coronary intervention: statin's advantage and the smoking 'paradox'. J Cardiovasc Pharmacol 2009; 53: 368-72.
27. Strauss MH, Hall AS. Angiotensin receptor blockers may increase risk of myocardial infarction unraveling the ARB-MI paradox. Circulation 2006; 114: 838-54.

28. Ge H, Zhou Y, Liu X, et al. Relationship between plasma inflammatory markers and platelet aggregation in patients with clopidogrel resistance after angioplasty. Angiology 2012; 63: 62-6.

29. Knight CJ, Panesar M, Wilson DJ, et al. Different effects of calcium antagonists, nitrates, and beta-blockers on platelet function. Possible importance for the treatment of unstable angina. Circulation 1997; 95: 125-32.

30. Salama MM, Morad AR, Saleh MA, et al. Resistance to low-dose aspirin therapy among patients with acute coronary syndrome in relation to associated risk factors. J Clin Pharm Ther 2012; 37: 630-6.

31. Liu XF, Cao J, Fan L, et al. Prevalence of and risk factors for aspirin resistance in elderly patients with coronary artery disease. J Geriatr Cardiol 2013; 10: 21-7.

32. Hobikoglu GF, Norgaz T, Aksu H, et al. Evaluation of aspirin resistance in patients with coronary artery disease. Türk Kardiyol Dern Arş Arch Turk Soc Cardiol 2005; 33: 212-6.

33. Akoglu H, Agbaht K, Piskinpasa S, et al. High frequency of aspirin resistance in patients with nephrotic syndrome. Nephrol Dial Transplant 2012; 27: 1460-6.

34. Kameda S, Sakata T, Kokubo Y, et al. Association of platelet aggregation with lipid levels in the japanese population: the suita study. J Atheroscler Thromb 2011; 18: 560-7.

35. Stejskal D, Vaclavik J, Lacnak B, et al. Aspirin resistance measured by cationic propyl gallate platelet aggregometry and recurrent cardiovascular events during 4 years of follow-up. Eur J Intern Med 2006; 17: 349-54.

36. Tanrikulu AM, Ozben B, Koc M, et al. Aspirin resistance in patients with chronic renal failure. J Nephrol 2011; 24: 636-46. 